\title{
Deux jours de Chambre médicale pour refaire le monde!
}

La Chambre médicale, qui est en quelque sorte le Parlement de la FMH, a tenu deux jours de séance les 18 et 19 mai derniers, et ... nous avons refait le monde: la FMH a maintenant de nouveaux statuts, qui devraient avoir de grandes répercussions sur son fonctionnement vis-à-vis de ses membres, et sur sa présence politique - et il ne s'agit pas là que de «wishful thinking»: les modifications apportées à notre organisation associative sont fondamentales et auront des effets majeurs sur la dynamique qui nous anime.

Je suis donc extrêmement reconnaissant à tous les participants à ces deux jours de session, et aux multiples groupements qu'ils représentent, d'avoir avec une très grande maturité politique accepté de parier sur l'avenir, sur une FMH plus représentative, plus souple et réactive, et pourquoi pas, plus imaginative.

De quoi s'agit-il? Les nouveautés les plus importantes sont:

- d'abord, la création d'une Assemblée des délégués de 33 membres, venant des sociétés cantonales et de spécialistes, ainsi que des divers «secteurs» du corps médical (assistants, médecins-chefs, femmes médecins, etc.); cette assemblée sera le vis-à-vis du Comité central, tous les deux mois environ, pour asseoir ses options stratégiques, les discuter et les valider - cela permettra à ce dernier de ne plus se trouver en vase clos comme trop souvent ces dernières années; il s'agit donc là d'une innovation fondamentale qui va profondément améliorer la présence politique du corps médical, et la qualité de cette présence;

- et ensuite, le poids politique accru donné à tout notre secteur «formation»; ce secteur sera plus autonome, par rapport au Comité central, dans la gestion des questions appartenant à sa sphère d'activités et dans sa prise de décisions, et il répondra le plus souvent directement de la Chambre médicale; le secteur «formation» est un domaine politiquement très sensible actuellement et il nous a paru important de faire droit à son besoin d'une image politique renforcée.
Par ailleurs, une «Commission de gestion» remplacera la Commission financière actuelle, inadaptée à tenir le rôle qui devrait être le sien dans une organisation de la taille de la nôtre; en outre, une limitation de durée des mandats a été introduite pour le Comité central, accompagnée d'incompatibilités claires avec des mandats économiques; et enfin, on a nettoyé les textes à droite et à gauche ...

Il faut aussi pour cette Chambre médicale relever trois autres points de l'ordre du jour qui méritent plus particulièrement de l'être:

- nous avons pris congé avec un peu de mélancolie d'Ursula Steiner-König, qui quitte le Comité central; à sa place, c'est Ernst Gähler (Appenzell) qui a été élu un portrait de lui paraîtra très bientôt dans le BMS, et nous lui souhaitons d'ores et déjà une cordiale bienvenue!

- Susi Stöhr a été élue vice-présidente; toutes nos félicitations!

- le budget 2007 a été voté - sans modification des cotisations mais avec un très gros déficit; nous pourrons absorber ce déficit en 2007, mais une réflexion va être menée pour préciser la politique budgétaire et financière de la FMH, en fonction des ressources possibles et bien sûr aussi des besoins croissants qui découlent de notre volume d'activité en constant développement.

Nous avons donc fait plusieurs pas de première importance lors de cette Chambre médicale; comme après toute bonne négociation, chacun s'est manifestement trouvé satisfait du résultat obtenu - preuve en soient l'unanimité (!) du vote final et les applaudissements qui l'ont ponctué.

En fait, nous n'aurons bientôt plus d'excuses pour ne pas être la FMH dont nous rêvons!

Jacques de Haller, président de la FMH 